\title{
Inhibitory effects of Chinese nutritional herbs in isogenic breast carcinoma cells with modulated estrogen receptor function
}

\author{
NITIN TELANG ${ }^{1}$, GUO LI $^{2}$, MEENA KATDARE ${ }^{3,4}$, DANIEL SEPKOVIC ${ }^{5}$, \\ LEON BRADLOW ${ }^{5}$ and GEORGE WONG ${ }^{2,6}$
}

\author{
${ }^{1}$ Cancer Prevention Research Program, Palindrome Liaisons Consultants, Montvale, NJ 07645-1559; \\ ${ }^{2}$ American Foundation for Chinese Medicine, New York, NY 1103-0905; ${ }^{3}$ Hampton University Skin of Color Research \\ Institute, Hampton, VA 23668; ${ }^{4}$ Department of Dermatology and Leroy T. Canoles Jr Cancer Research Center, \\ Eastern Virginia Medical School, Norfolk, VA 23507; ${ }^{5}$ Hackensack University Medical Center, Hackensack, NJ 07601; \\ ${ }^{6}$ Department of Integrative Medicine, Mount Sinai Beth Israel Medical Center, New York, NY 10003, USA
}

Received March 3, 2016; Accepted July 7, 2016

DOI: $10.3892 / \mathrm{ol} .2016 .5197$

\begin{abstract}
In estrogen receptor (ER) $)^{+}$MCF-7 cells, ER represents a ligand-activated transcription factor, and 17ß-estradiol (E2) represents its physiological ligand. Maintenance of the human breast carcinoma-derived MCF-7 cells with $0.7 \%$ serum selected a proliferative sub-population of E2-responsive cells with transiently non-functional ER due to limited availability of E2. Culture of MCF-7 cells in the presence of either 0.7\% serum, $<1 \mathrm{nM}$ E2 or $0.7 \%$ serum $+20 \mathrm{nM} \mathrm{E2}$ selected isogenic cells with either non-functional ER (ER-NF) or functional ER (ER-F) phenotype. The two phenotypes responded to the growth-promoting effects of E2 and to the growth-inhibitory effects of the selective ER modulator tamoxifen, indicating retention of E2 responsiveness. Comparative dose-response experiments with Chinese nutritional herbs on ER-NF and ER-F cells identified the inhibitory concentration (IC) $)_{50}$ values for these herbs, while the $\mathrm{IC}_{50}$ ratios for the ER-NF:ER-F phenotypes facilitated their rank ordering in terms of efficacy. Out of the 11 efficacious herbs tested, five herbs exhibited ER-F > ER-NF inhibitory activity, four exhibited ER-F = ER-NF inhibitory activity and two exhibited ER-NF > ER-F inhibitory activity. Extracts from representative herbs, Lycium barbarum bark, Epimedium grandiflorum and Cornusofficinalis, fromeach of the three groups inhibited anchorage-independent growth, induced G1 or G2/M arrest and/or apoptosis, and generated anti-proliferative E2 metabolites. The differential growth inhibition in ER-NF and ER-F phenotypes, together with the mechanistic efficacy of representative herbs, identified potential leads for their efficacy on $\mathrm{ER}^{+}$and/or ER' breast cancer.
\end{abstract}

Correspondence to: Dr Nitin Telang, Cancer Prevention Research Program, Palindrome Liaisons Consultants, 10 Rolling Ridge Road, Suite B, Montvale, NJ 07645-1559, USA

E-mail: entitytoo@gmail.com; ntelang3@gmail.com

Key words: isogenic breast carcinoma cells, nutritional herbs, growth inhibition

\section{Introduction}

Hormone-responsive estrogen receptor $(\mathrm{ER})^{+}$clinical breast cancer responds to endocrine therapy involving the use of selective ER modulators and selective inhibitors of estradiol biosynthesis $(1,2)$. However, long-term treatment with these agents is frequently associated with acquired tumor resistance, resulting in limited efficacy that confers a negative impact on patient response (1-3). These aspects underscore the importance of identifying novel therapeutic agents with improved efficacy and low systemic toxicity.

Chinese nutritional herbs are widely used in the management of general health issues in women as well as an alternative treatment option for breast cancer (4-7). Naturally occurring herbal preparations may exhibit an acceptable toxicity profile, and thus are likely to favorably interact with conventional endocrine therapy to reduce toxicity and enhance efficacy of pharmacological therapeutic agents. However, the impact of herb-drug interaction on therapeutic efficacy remains unknown (4).

The estrogen-dependent human breast carcinoma-derived $\mathrm{ER}^{+} \mathrm{MCF}-7$ cell line represents a well-established preclinical cell culture system for hormone-responsive clinical breast cancer. MCF-7 cells depend on 17ß-estradiol (E2) for cell proliferation in vitro and for tumor development in vivo, and thereby represent an important model for evaluating the efficacy of novel agents that function via modulation of the cellular and molecular response to estrogens (8). ER functions as a ligand-activated nuclear transcription factor, with E2 as the endogenous ligand, to promote cellular proliferation and tumorigenic growth via a complex signaling cascade that involves the coordinated functions of several co-activators and co-repressors, resulting in the expression of selected estrogen-responsive target genes $(2,3,8)$. Sub-physiological concentrations of E2 render ER as a transiently non-functional receptor $(3,8)$.

Our previous studies have demonstrated that MCF-7 cells adapted for growth in chemically defined serum-depleted culture conditions retain their responsiveness to E2, and respond to the growth-inhibitory effects of several 
mechanistically distinct pharmacological agents and selected herbal extracts (9-13).

In traditional Chinese medicine, combinations of several nutritional herbs are commonly used as a palliative treatment option for breast cancer patients $(5,7)$. The herbs selected in the present study represent some of these clinically relevant herbs. In the present study, isogenic MCF-7 phenotypes with modulated ER function were isolated, and these models were used to compare the growth-inhibitory efficacy of the selected Chinese nutritional herbs. The outcome of the present study has provided data to demonstrate the differential efficacy of herbs depending upon the functional status of ER, thus identifying potential leads to prioritize efficacious herbs for secondary prevention and/or for therapy of $\mathrm{ER}^{+}$and $\mathrm{ER}^{-}$clinical breast cancer.

\section{Materials and methods}

Cell lines. The parental ER $\mathrm{MCF}^{+} 7$ cell line was originally obtained from the Michigan Cancer Foundation (Detroit, MI, USA). These cells were cultured in Dulbecco's modified Eagle-F12 medium supplemented with $10 \%$ heat inactivated fetal calf serum (Gibco; Thermo Fisher Scientific, Inc., Waltham, MA, USA), $10 \mu \mathrm{g} / \mathrm{ml}$ insulin (Eli Lilly and Company, Indianapolis, IN, USA) and $1 \mu \mathrm{M}$ dexamethasone (Sigma-Aldrich, St. Louis, MO, USA), following published protocols $(9,10)$.

Isolation and characterization of non-functional ER (ER-NF) and functional ER (ER-F) phenotypes. To isolate and characterize the ER-NF phenotype, parental MCF-7 cells were adapted for growth in medium supplemented with decreasing serum concentrations $(7.5,5.0,2.5,1.0$ and $0.7 \%)$, corresponding to decreased levels of E2 ranging from $15 \mathrm{nM}$ to $<1 \mathrm{nM}$. The cells from the $0.7 \%$ serum group $(\mathrm{E} 2<1 \mathrm{nM})$ were continuously maintained in medium supplemented with $0.7 \%$ serum for $\geq 5$ passages. These cells were tested for their E2 response by monitoring their growth in the presence of E2 at 1, 5, 10 and $20 \mathrm{nM}$ concentrations. The stock cultures of ER-NF cells were routinely maintained in medium supplemented with $0.7 \%$ serum $(\mathrm{E} 2<1 \mathrm{nM})$. The ER-NF phenotype is defined as the cell population that exhibits progressive growth in the presence of chemically defined serum-depleted medium (serum, 0.7\%; E2 <1 nM).

To isolate the ER-F phenotype, ER-NF cells were maintained in serum-depleted medium $(0.7 \%$ serum $)+20 \mathrm{nM} \mathrm{E2}$ for $\geq 5$ passages, and the stock cultures were maintained in E2-supplemented medium. Routinely, the ER-NF and ER-F phenotypes were maintained in a humidified atmosphere of $95 \%$ air and $5 \% \mathrm{CO}_{2}$, and were sub-cultured at a 1:4 ratio when $80 \%$ confluent. The ER-F phenotype is defined as the cell population that exhibits progressive growth in the presence of a physiologically relevant concentration of E2.

To further characterize E2 responsiveness, the effect of the selective ER modulator tamoxifen (TAM) was evaluated on the ER-NF and ER-F phenotypes by monitoring population-doubling time, saturation density and anchorage-independent (AI) colony formation $(12,13)$. For these experiments, the stock solutions of E2 and TAM (both from Sigma-Aldrich) were prepared in $100 \%$ ethanol (Thermo
Fisher Scientific, Inc.) at a $100 \mathrm{mM}$ concentration, and were serially diluted in the culture medium to obtain the final concentrations of $20 \mathrm{nM}$ E2 and $20 \mathrm{nM}$ TAM employed for the treatment of the cell cultures.

Preparation of herbal extracts. The Chinese nutritional herbs used in the present study were provided by Professor G.Y.C Wong (American Foundation for Chinese Medicine, New York, NY, USA). The Chinese nutritional herbs selected for the present study include Angelica sinensis (AS), Cibotium barometz (CB), Cornus officinalis (CO), Cuscuta sinensis (CS), Dipsacus asperoides (DA), Epimedium grandiflorum (EG), Eucommia ulmoides (EU), Ligusticum chuanxiong (LC), Ligustium lucidum (LL), Lycium barbarum bark (LBB), Lycium barbarum fruit (LBF), Morinda officinalis (MO) and Psoralea corylifolia (PC). The sources of herbal material for the preparation of aqueous extracts from these nutritional herbs are presented in Table I. The selection of the specific parts of the nutritional herbs and the method to prepare the extracts for the present study were based on the protocols followed in traditional Chinese medicine $(5,7)$.

To prepare non-fractionated aqueous extracts, $20 \mathrm{~g}$ of the herbal materials were boiled in $200 \mathrm{ml}$ of distilled water until the volume was reduced to $100 \mathrm{ml}$ (extract \#1). The residue from extract \#1 was then boiled in $100 \mathrm{ml}$ of distilled water until the volume was reduced to $50 \mathrm{ml}$ (extract \#2). Extracts \#1 and \#2 were combined (total volume, $150 \mathrm{ml}$ ) and boiled until the volume was reduced to $25 \mathrm{ml}$. These combined extracts were centrifuged $(500 \mathrm{x} \mathrm{g}$ at room temperature for $10 \mathrm{~min}$ ), and the final aqueous supernatant $(20 \mathrm{ml})$ was collected. This non-fractionated aqueous supernatant served as the stock solution $(100 \%)$, which was serially diluted with the culture medium to obtain the final concentrations of $2,1,0.5,0.05$, 0.02 and $0.01 \%$ used for the treatment of the cell cultures.

Dose-response experiments. For dose-response experiments to identify the inhibitory concentration (IC) $)_{50}$ values of the different Chinese herbs, the ER-NF and ER-F phenotypes were treated with the herbal extracts at concentrations ranging from 2 to $0.01 \%$. The cells were plated at an initial seeding density of $1.0 \times 10^{5}$ cells per T-25 flask. After a 24-h attachment period, the cultures were treated with the corresponding diluted herbal extracts for 7 days. At the end of the 7 th day of treatment, the cells were trypsinized, and the cell number and viability were determined by the trypan blue exclusion test (Sigma-Aldrich).

These dose-response experiments provided data regarding the $\mathrm{IC}_{50}$ values of the different herbs for the ER-NF and ER-F phenotypes, while the ER-NF:ER-F ratio determined from the above $\mathrm{IC}_{50}$ provided the rank order for the growth-inhibitory efficacy of the herbs tested. Additionally, data obtained from the dose-response experiments identified the maximum cytostatic concentrations $\left(\mathrm{IC}_{90}\right)$ of the individual herbal extracts, and were used to distinguish the maximum cytostatic effects from the toxic effects. The $\mathrm{IC}_{90}$ was defined as the highest concentration of the herbal extract that results in a surviving cell population greater than, or equal to, the initial seeding density, while a surviving population of less than that of the initial seeding density was considered as treatment-associated toxic response. 
Table I. Chinese nutritional herbs.

\begin{tabular}{lcc}
\hline Botanical name & Abbreviation & Source of extract \\
\hline Angelica sinensis & AS & Root \\
Cibotium barometz & CB & Root \\
Cornus officinalis & CO & Fruit \\
Cuscuta sinensis & CS & Seed \\
Dipsacus asperoides & DA & Root \\
Epimedium grandiflorum & EG & Leaf, stem \\
Eucommia ulmoides & EU & Bark \\
Ligusticum chuanxiong & LC & Root \\
Ligustrum lucidum & LL & Fruit \\
Lycium barbarum bark & LBB & Bark \\
Lycium barbarum fruit & LBF & Fruit \\
Morinda officinalis & MO & Root \\
Psoralea corylifolia & PC & Seed \\
\hline
\end{tabular}

AI growth assay. The protocol for the AI growth assay has been optimized and published $(12,13)$. For the assay, ER-NF cells were suspended in $0.33 \%$ agar (Sigma-Aldrich) at a density of 1,000 cells/2 ml/well in 6-well plates. The treatment groups contained the herbal extracts at their respective $\mathrm{IC}_{90}$ value in the cell suspension prepared in $0.33 \%$ agar. The cell suspension was overlaid on a basement layer of $0.66 \%$ agar, and the cultures were incubated at $37^{\circ} \mathrm{C}$ for 21 days in a humidified atmosphere of $95 \%$ air and $5 \% \mathrm{CO}_{2}$. The non-adherent AI colonies that developed in the suspension cultures were counted at the end of the treatment.

Cell cycle progression and cellular apoptosis. To examine the effects of the herbal extracts on cell cycle progression and cellular apoptosis, the control and treated cultures were analyzed by flow cytometry using a previously published protocol (14). Briefly, trypsinized cell cultures from the control and treated groups were stained with propidium iodide (Calbiochem; EMD Millipore, Billerica, MA, USA), and the percentage of cell population in the $\mathrm{G} 1, \mathrm{~S}, \mathrm{G} 2 / \mathrm{M}$ and sub-G0 (apoptotic) phases of the cell cycle were monitored using the EPICS 752 flow cytometer (Beckman Coulter, Inc., Brea, CA, USA), which was equipped with 488-nM excitation and 630-nM emission long-pass filters. The cell cycle phase distribution was analyzed using the MultiCycle MPLUS software (Phoenix Flow Systems, San Diego, CA, USA). The data were expressed as percentage of cell population in each phase of the cell cycle, as well as G1:S+G2/M ratio.

Cellular metabolism of E2. To examine the effects of the herbal extracts on the cellular metabolism of E2, the medium from the control and treated cultures after 48-h incubation was analyzed for the presence of selected E2 metabolites, including estrone (E1), 2-hydroxyestrone (2-OHE1), 16 $\alpha$-hydroxyestrone (16 $\alpha$-OHE1) and estriol (E3), on a $6980 \mathrm{~N}$ gas chromatograph (Agilent Technologies, Inc., Santa Clara, CA, USA) equipped with a 5973 mass selective detector (MSD) (Agilent Technologies, Inc.), a 7683 injector (Agilent Technologies, Inc.) and a HPGI 701CA MSD Chemstation (Agilent Technologies,
Inc.), using a previously published gas chromatography-mass spectrometry-based assay $(10,12,13)$. The data were expressed as 2-OHE1:16 $\alpha-\mathrm{OHE1}$ and $\mathrm{E} 3: 16 \alpha-\mathrm{OHE} 1$ ratios.

Statistical analysis. The experiments for dose response, population-doubling time and saturation density were performed with $n=6$ flasks per treatment group. The AI growth assay was performed with $n=18$ wells per treatment group, while the experiments for cell cycle analysis, cellular apoptosis and E2 metabolism were performed using n=3 flasks per treatment group for each assay.

The statistical significance of the differences in mean \pm standard deviation (SD) values between the data points from the control and the experimental groups for the individual experiments was analyzed by a two-sample $t$-test using GraphPad Prism software version 5.0 (GraphPad Software, Inc., La Jolla, CA, USA). $\mathrm{P}<0.05$ was considered to indicate a statistically significant difference.

\section{Results}

Isolation and characterization of ER-NF and ER-F phenotypes. The data presented in Fig. 1A demonstrates that the parental MCF-7 cells exhibit a progressive decrease in the number of surviving cells as a function of decreased serum concentration. Thus, relative to the $10 \%$ serum group, the $5.0 \%$ and $2.5 \%$ serum groups exhibited a $43.7 \%(\mathrm{P}=0.04)$ and a $53.1 \%(\mathrm{P}=0.04)$ decrease, respectively, and the $1.0 \%$ serum group exhibited a $92.2 \%$ decrease $(\mathrm{P}=0.03)$ in the surviving cell population. The cells from the $1.0 \%$ serum group are also effectively adapted to progressive growth in the presence of $0.7 \%$ serum. The data presented in Fig. 1B demonstrates that the cells maintained in the presence of $0.7 \%$ serum exhibit a progressive increase in the number of viable cells as a function of increasing concentrations of E2. Relative to the $<1 \mathrm{nM}$ E2 group, cells treated with $5 \mathrm{nM}$ E2 exhibited an $83.3 \%$ increase $(\mathrm{P}=0.03)$, and those treated with $10 \mathrm{nM}$ E2 and $20 \mathrm{nM}$ E2 exhibited a 1.2-fold $(\mathrm{P}=0.01)$ and a 1.4 -fold $(\mathrm{P}=0.01)$ increase, respectively, in the surviving population. Thus, the surviving population from the $0.7 \%$ serum group $(\mathrm{E} 2<1 \mathrm{nM})$ represents the ER-NF phenotype, while that from the $0.7 \%$ serum + 20 nM E2 group represents the ER-F phenotype.

The data presented in Table II examines the effect of TAM on E2-promoted growth in the ER-NF and ER-F phenotypes. The ER-NF phenotype, in response to treatment with E2 + TAM, exhibits a $29.1 \%$ increase $(\mathrm{P}=0.05)$ in population-doubling time, a $59.1 \%$ decrease $(\mathrm{P}=0.04)$ in saturation density and a $59.3 \%$ decrease $(\mathrm{P}=0.04)$ in the number of $\mathrm{AI}$ colonies, relative to the E2-treated control. Similarly, in the ER-F phenotype, treatment with $\mathrm{E} 2+\mathrm{TAM}$ induces a $26.5 \%$ increase $(\mathrm{P}=0.05)$ in the population doubling time, a $65.5 \%$ decrease $(\mathrm{P}=0.04)$ in the saturation density and a $62.1 \%$ decrease $(\mathrm{P}=0.04)$ in the number of AI colonies, relative to the E2-treated control. Thus, these data demonstrate that TAM antagonizes the growth-promoting effect of E2 in the ER-NF and ER-F phenotypes.

Effects of herbal extracts on ER-NF and ER-F phenotypes. The data on growth inhibition of ER-NF and ER-F phenotypes by herbal extracts are presented as $\mathrm{IC}_{50}$ values in Tables III and IV, respectively. The rank order of preferential growth-inhibitory 


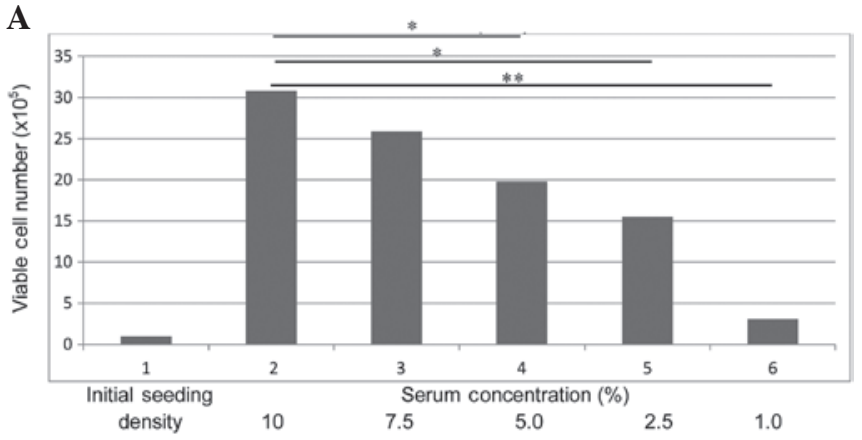

B

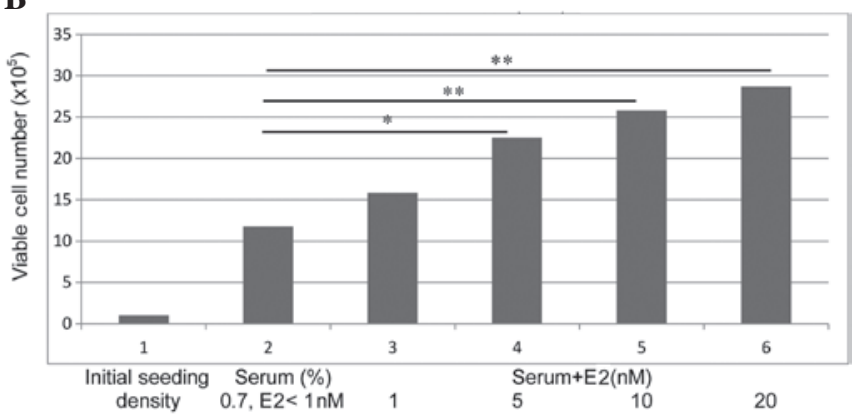

Figure 1. (A and B) Growth of human mammary carcinoma-derived MCF-7 cells. (A) Adaptation for growth in chemically defined serum-depleted culture medium. A progressive decrease in the viable cell number as a function of decreased serum concentration was noted. Significant results: 10 vs. $5 \%$ serum ( $(\mathrm{P}=0.04) ; 10$ vs. $2.5 \%$ serum $\left({ }^{*} \mathrm{P}=0.04\right) ; 10$ vs. $1.0 \%$ serum ( $\left.{ }^{* *} \mathrm{P}=0.03\right)$. (B) Response of MCF-7 cells maintained in the culture medium supplemented with $0.7 \%$ serum and $\mathrm{E} 2$ concentrations ranging from $<1 \mathrm{nM}$ to physiologically relevant levels. A progressive increase in the viable cell number as a function of increasing concentrations of $\mathrm{E} 2$ was noted. Significant results: $<1$ vs. $5 \mathrm{nM} \mathrm{E} 2\left({ }^{*} \mathrm{P}=0.03\right) ;<1$ vs. $10 \mathrm{nM} \mathrm{E} 2\left({ }^{* *} \mathrm{P}=0.01\right) ;<1$ vs. $20 \mathrm{nM} \mathrm{E} 2$ $\left.{ }^{* * *} \mathrm{P}=0.01\right)$. E2, 17ß-estradiol.

effects of herbal extracts on the ER-NF and ER-F phenotypes are presented as the $\mathrm{IC}_{50}$ ratio of the ER-NF vs. ER-F phenotype (Table $\mathrm{V})$. These data revealed that an $\mathrm{IC}_{50}$ ratio of $>1$ was exhibited by LBB, EU, LBF, PC and DA, thus representing preferential efficacy towards the ER-F phenotype. $\mathrm{An}^{\mathrm{IC}_{50}}$ ratio of 1 was exhibited by LL, CS, EG and LC, thus representing equal efficacy towards the ER-NF and ER-F phenotypes, while an $\mathrm{IC}_{50}$ ratio of $<1$ was exhibited by $\mathrm{AS}$ and $\mathrm{CO}$, thus representing preferential efficacy towards the ER-NF phenotype. The extract prepared from CB exhibited a non-significantly different 8 and 16\% growth inhibition for the ER-NF and ER-F phenotypes, respectively, at the highest concentration tested $(2.0 \%)$. Similarly, the extract prepared from MO exhibited a non-significantly different 9.7 and $8.5 \%$ growth inhibition for the two phenotypes, respectively, at the equivalent highest concentration. Thus, the extracts from CB and MO were demonstrated to be essentially ineffective in the present experimental system.

Based on the data from the rank order of effective $\mathrm{IC}_{50}$ values in the ER-NF and ER-F phenotypes, herbal extracts from LBB (which demonstrated preferential efficacy for ER-F cells), EG (which demonstrated identical efficacy in ER-NF and ER-F cells) and CO (which demonstrated preferential efficacy for ER-NF cells), were examined for their effect on AI growth in MCF-7 cells. The data presented in Fig. 2, which are expressed as the number of colonies [mean $\pm \mathrm{SD}$, $\mathrm{n}=18 /$ treatment group) demonstrate that MCF-7 cells maintained in the presence of $0.7 \%$ serum $(\mathrm{E} 2<1 \mathrm{nM})$ plus a physiologically relevant concentration of $20 \mathrm{nM}$ E2 exhibit a $116.3 \%$ increase $(\mathrm{P}=0.01)$ in the number of AI colonies (mean AI colony number, $36.7 \pm 2.4$ ), relative to that observed in the $0.7 \%$ serum-treated control group (mean AI colony number, 17.2 \pm 3.4 ). Furthermore, LBB treatment resulted in a mean AI colony number of $4.5 \pm 1.3$, while treatment with EG and $\mathrm{CO}$ led to a mean AI colony number of $4.9 \pm 2.5$ and $4.7 \pm 2.4$, respectively. Thus, extracts from $\mathrm{LBB}, \mathrm{EG}$ and $\mathrm{CO}$ at their respective $\mathrm{IC}_{90}$ value produced a $>80 \%$ decrease $(\mathrm{P}=0.02)$ in the number of E2-promoted AI colonies.

Mechanisms for efficacy of selected herbal extracts. The data on the effect of extracts from LBB, EG and CO on the phases of the cell cycle are presented in Table VI. The LBB extract induced a $48.2 \%$ reduction $(\mathrm{P}=0.04)$ in the $\mathrm{S}$-phase population and a 1.5 -fold increase $(\mathrm{P}=0.01)$ in the $\mathrm{G} 2 / \mathrm{M}$-phase population, leading to $\mathrm{G} 2 / \mathrm{M}$-phase arrest. In contrast, treatment with EG resulted in a $55.1 \%$ increase $(\mathrm{P}=0.04)$ in the population in $\mathrm{G} 1$ phase and a $83.9 \%$ decrease $(\mathrm{P}=0.02)$ in the G2/M-phase population. Treatment with $\mathrm{CO}$ resulted in a $33.5 \%$ increase $(P=0.04)$ in the number of cells in the $\mathrm{G} 1$ phase and a $45.9 \%$ decrease $(\mathrm{P}=0.04)$ in that in the $\mathrm{S}$ phase. Thus, these data demonstrate that the above three extracts counteract the growth-promoting effect of E2 via their specific effects on distinct phases of the cell cycle, leading to an altered $\mathrm{G} 1: \mathrm{S}+\mathrm{G} 2 / \mathrm{M}$ ratio. Thus, the data on the cell cycle progression, expressed as $\mathrm{G} 1: \mathrm{S}+\mathrm{G} 2 / \mathrm{M}$ ratio (mean $\pm \mathrm{SD}$, $\mathrm{n}=3 /$ treatment group), which was obtained using extracts from the selected herbs LBB, EG and CO (Fig. 3A), demonstrated that, compared with the control, which exhibited a G1:S+G2/M ratio of $1.02 \pm 0.3$, the extract from $\mathrm{LBB}$ at its $\mathrm{IC}_{90}$ value led to a ratio of $0.8 \pm 0.1$, which represents a $25.5 \%$ reduction $(\mathrm{P}=0.04)$. In contrast, the extract from $\mathrm{EG}$ at its $\mathrm{IC}_{90}$ value induced a 2.4-fold increase (ratio, 3.5 $\pm 1.1 ; \mathrm{P}=0.02$ ), while the extract from CO induced a $98.0 \%$ increase (ratio, 2.02 $\pm 0.6 ; \mathrm{P}=0.02$ ).

The data on the status of cellular apoptosis presented in Fig. 3B demonstrate that, compared with the control (sub-G0 population, $1.9 \pm 0.5 \%$ ), the extract from LBB induced a 2.1-fold increase (sub-G0 population, $5.9 \pm 1.3 \%$; $\mathrm{P}=0.01$ ). Additionally, fluorescence microscopy of these apoptotic cells stained with the ApopTag Fluorescein In Situ Apoptosis Detection kit (Merck Millipore, Darmstadt, Germany) exhibited nuclear fragmentation corresponding to the extent of apoptosis, as detected by the percentage of sub-G0 population (data not shown). By contrast, extracts from EG (sub-G0 population, $1.6 \pm 0.9 \%$ ) and $\mathrm{CO}$ (sub-G0 population, $1.4 \pm 0.9 \%$ ) were essentially ineffective in inducing cellular apoptosis. These data were not significantly different from the control group.

The data from the experiments on the cellular metabolism of E2 are presented as 2-OHE1:16 $\alpha-\mathrm{OHE1}$ ratio (mean $\pm \mathrm{SD}$, $\mathrm{n}=3$ /treatment group) in Fig. $4 \mathrm{~A}$ and as E3:16 $\alpha-\mathrm{OHE} 1$ ratio in Fig. 4B. Relative to the control (ratio, 0.5 \pm 0.1 ), extracts

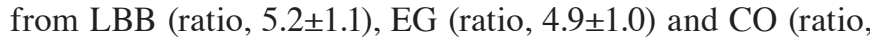
$6.8 \pm 1.4$ ), induced a 9.2, 8.8 and 12.6-fold increase, respectively $(\mathrm{P}=0.01)$, in the 2-OHE1:16 $\alpha-\mathrm{OHE1}$ ratio (Fig. 4A).

Regarding the E3:16 $\alpha$-OHE1 ratio (Fig. 4B), relative to the control (ratio, $0.1 \pm 0.04$ ), the LBB extract exhibited a ratio of $0.7 \pm 0.3$, while the extract from EG exhibited a ratio of $0.6 \pm 0.2$ and that from $\mathrm{CO}$ exhibited a ratio of $0.4 \pm 0.2$, thus inducing a 6,5 and 3 -fold increase, respectively $(\mathrm{P}=0.01)$. 
Table II. Combinatorial effects of E2 and TAM on ER-NF and ER-F phenotypes.

\begin{tabular}{lrrrrr}
\hline & \multicolumn{3}{c}{ ER-NF } & & ER-F \\
\cline { 2 - 5 } Biomarker & E2 & E2+TAM & & E2 & \\
\hline Population-doubling time (h) $^{\mathrm{a}}$ & $27.8 \pm 1.7$ & $35.9 \pm 1.9^{\mathrm{d}}$ & & $27.2 \pm 1.4$ & $34.4 \pm 1.4^{\mathrm{e}}$ \\
Saturation density (x10 cells) $^{\mathrm{b}}$ & $18.1 \pm 0.3$ & $7.4 \pm 0.5^{\mathrm{f}}$ & & $26.1 \pm 1.8$ & $9.0 \pm 1.6^{\mathrm{g}}$ \\
AI colonies (no.) $^{\mathrm{c}}$ & $36.9 \pm 2.1$ & $15.0 \pm 1.6^{\mathrm{h}}$ & & $37.2 \pm 2.1$ & $14.1 \pm 1.0^{\mathrm{i}}$ \\
\hline
\end{tabular}

${ }^{\mathrm{a} C a l c u l a t e d}$ from the exponential growth phase of cultures treated with $20 \mathrm{nM}$ E2 or equimolar concentrations of E2 + TAM. Data are rep-

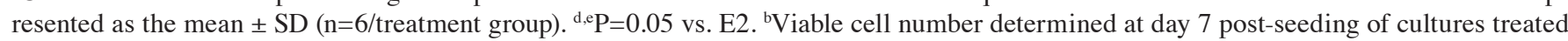
with $20 \mathrm{nM}$ E2 or equimolar concentrations of E2 + TAM. Data are represented as the mean $\pm \mathrm{SD}$ ( $\mathrm{n}=6 /$ treatment group). ${ }^{\mathrm{f}, \mathrm{g}} \mathrm{P}=0.04 \mathrm{vs}$. E2. ${ }^{\mathrm{c}}$ Determined at day 21 post-seeding of cultures treated with $20 \mathrm{nM} \mathrm{E} 2$ or equimolar concentrations of E2 + TAM. Data are represented as the mean $\pm \mathrm{SD}$ ( $\mathrm{n}=12 /$ treatment group). ${ }^{\mathrm{h}, \mathrm{i}} \mathrm{P}=0.04 \mathrm{vs}$. E2. ER-NF, estrogen receptor non-functional; ER-F, estrogen receptor functional; E2, 17ß-estradiol; TAM, tamoxifen; AI, anchorage-independent; SD, standard deviation.

Table III. Rank order of efficacy by $\mathrm{IC}_{50}$ of Chinese nutritional herbs on the estrogen receptor non-functional phenotype.

\begin{tabular}{|c|c|}
\hline Herbal extract & $\mathrm{IC}_{50}(\%)^{\mathrm{a}}$ \\
\hline LBB & 0.01 \\
\hline $\mathrm{CO}$ & 0.03 \\
\hline DA & 0.19 \\
\hline EU & 0.23 \\
\hline LL & 0.30 \\
\hline $\mathrm{PC}$ & 0.40 \\
\hline EG & 0.46 \\
\hline $\mathrm{CS}$ & 0.60 \\
\hline $\mathrm{LC}$ & 0.60 \\
\hline AS & 0.70 \\
\hline $\mathrm{LBF}$ & 0.90 \\
\hline $\mathrm{CB}$ & Not reached ${ }^{b}$ \\
\hline MO & Not reached ${ }^{\mathrm{b}}$ \\
\hline \multicolumn{2}{|c|}{$\begin{array}{l}\text { aDetermined after a } 7 \text {-day treatment with the herbal extract. Estrogen } \\
\text { receptor non-functional MCF-7 cells were maintained in culture } \\
\text { medium supplemented with } 0.7 \% \text { serum }\left(<1 \mathrm{nM} 17 \beta \text {-estradiol). }{ }^{b} \mathrm{IC}_{50}\right. \\
\text { was not achieved at the concentration range of } 0.01-2.00 \% \text {. IC, inhib- } \\
\text { itory concentration. AS, Angelica sinensis; CB, Cibotium barometz; } \\
\mathrm{CO} \text {, Cornus officinalis; CS, Cuscuta sinensis; DA, Dipsacus asper- } \\
\text { oides; EG, Epimedium grandiflorum; EU, Eucommia ulmoides; LC, } \\
\text { Ligusticum chuanxiong; LL, Ligustium lucidum; LBB, Lycium bar- } \\
\text { barum bark; LBF, Lycium barbarum fruit; MO, Morinda officinalis; } \\
\text { PC, Psoralea corylifolia. }\end{array}$} \\
\hline
\end{tabular}

\section{Discussion}

Global gene expression profiling of clinical breast cancer has provided improved molecular/genetic classification of cancer subtypes, and thereby, has facilitated rational subtype-targeted therapy (15). Similar classification of commercially available breast carcinoma cell lines has refined the applicability of these human tissue-derived preclinical models for specific molecular subtypes (16-18). It is well established that long-term treatment with endocrine therapy is frequently associated with de novo or acquired resistance via deregulation of multiple
Table IV. Rank order of efficacy by $\mathrm{IC}_{50}$ of Chinese nutritional herbs on the estrogen receptor functional phenotype.

\begin{tabular}{lc}
\hline Herbal extract & $\mathrm{IC}_{50}(\%)^{\mathrm{a}}$ \\
\hline LBB & 0.001 \\
CO & 0.070 \\
EU & 0.090 \\
DA & 0.130 \\
PC & 0.260 \\
LL & 0.280 \\
LBF & 0.390 \\
EG & 0.440 \\
CS & 0.570 \\
LC & 0.620 \\
AS & 1.170 \\
\hline
\end{tabular}

aDetermined after a 7-day treatment with the herbal extract. Estrogen receptor functional MCF-7 cells were maintained in culture medium supplemented with $0.7 \%$ serum and $20 \mathrm{nM}$ $17 \beta$-estradiol. IC, inhibitory concentration. AS, Angelica sinensis; CO, Cornus officinalis; CS, Cuscuta sinensis; DA, Dipsacus asperoides; EG, Epimedium grandiflorum; EU, Eucommia ulmoides; LC, Ligusticum chuanxiong; LL, Ligustium lucidum; LBB, Lycium barbarum bark; LBF, Lycium barbarum fruit; PC, Psoralea corylifolia.

genetic/molecular pathways that are critical for proliferation and survival signaling, and may lead to compromised therapeutic efficacy (3). Reliable cell culture models that facilitate the identification of cellular/molecular pathways of therapeutic resistance should provide valuable experimental approaches for screening of potential lead compounds that are efficacious against therapy-resistant breast cancer.

The cell culture models for $\mathrm{ER}^{+} /$progesterone receptor $(\mathrm{PR})^{+}$ /human epidermal growth factor (HER-2)- luminal A and $\mathrm{ER}^{-} / \mathrm{PR}^{-} / \mathrm{HER}-2^{-}$triple negative subtypes have been commonly used as comparative experimental systems for $\mathrm{ER}^{+}$and $\mathrm{ER}^{-}$ clinical breast cancer, respectively $(2,8,16)$. Specific genetic differences in these two models other than the status of ER expression have been documented (16-18). These intrinsic 
Table V. Comparative efficacy by $\mathrm{IC}_{50}$ of Chinese nutritional herbs on the isogenic ER-NF and ER-F phenotypes.

\begin{tabular}{lc}
\hline Herbal extract & ER-NF:ER-F ratio $\left(\mathrm{IC}_{50}\right)^{\mathrm{a}}$ \\
\hline LBB & 10.00 \\
EU & 2.56 \\
LBF & 2.31 \\
PC & 1.54 \\
DA & 1.46 \\
LL & 1.07 \\
CS & 1.05 \\
EG & 1.04 \\
LC & 0.97 \\
AS & 0.59 \\
CO & 0.43
\end{tabular}

${ }^{\mathrm{a}} \mathrm{IC}_{50}$ ratio $>1$, preferential efficacy for the ER-F phenotype; $\mathrm{IC}_{50}$ ratio $=1$, equivalent efficacy for the ER-NF and ER-F phenotypes; $\mathrm{IC}_{50}$ ratio $<1$, preferential efficacy for the ER-NF phenotype. ER-NF, estrogen receptor non-functional; ER-F, estrogen receptor functional; IC, inhibitory concentration; AS, Angelica sinensis; CO, Cornus officinalis; CS, Cuscuta sinensis; DA, Dipsacus asperoides; EG, Epimedium grandiflorum; EU, Eucommia ulmoides; LC, Ligusticum chuanxiong; LL, Ligustium lucidum; LBB, Lycium barbarum bark; LBF, Lycium barbarum fruit; PC, Psoralea corylifolia.

molecular/genetic differences may limit the utility of these models for comparative investigations. Availability of isogenic phenotypes that are genetically identical but differ only in ER function should facilitate stringently controlled comparative studies. Additionally, the present comparative study on ER-NF and ER-F phenotypes provides an experimental approach that simulates a situation in the progression of clinical breast cancer where relapse is due to a change in ER status from positive to negative (3).

The present approach on the human mammary carcinoma-derived MCF-7 cells, which served as a model for the breast cancer luminal A subtype $(8,16,17)$, has provided isogenic cell phenotypes with modulated ER function. Long-term growth adaptation of the parental MCF-7 cells in chemically defined serum-depleted medium $(0.7 \%$ serum, E2 $<1 \mathrm{nM}$ ) selected the transient ER-NF phenotype due to limited availability of a physiologically relevant concentration of E2. The ER-NF phenotype continued to exhibit progressive growth in the presence of E2 within the physiologically achievable range. Additionally, the growth-promoting effect of E2 was abrogated in the presence of the selective ER modulator TAM. Collectively, these observations provided evidence that the ER-NF and ER-F phenotypes effectively retain E2 responsiveness.

The ER-NF cells maintained in the presence of $0.7 \%$ serum $(\mathrm{E} 2<1 \mathrm{nM})$ and the ER-F cells maintained in the presence of $0.7 \%$ serum $+20 \mathrm{nM} \mathrm{E2}$ provided the isogenic models for the present study to compare the growth-inhibitory efficacy of extracts from selected Chinese nutritional herbs. Although these ER-NF and ER-F isogenic phenotypes adequately facilitated preliminary screening of efficacious herbs, both these

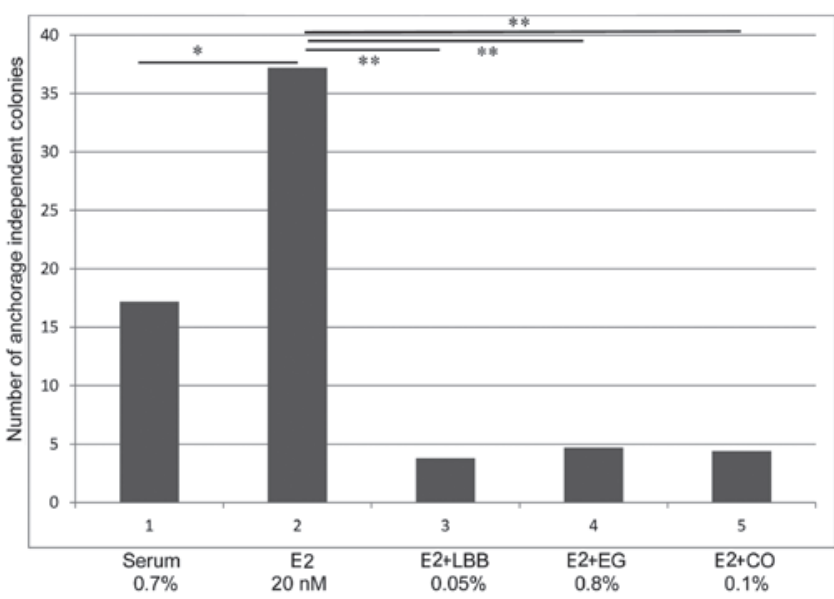

Figure 2. Inhibition of AI growth in human mammary carcinoma-derived MCF-7 cells. E2 promoted the number of AI colonies and the E2-promoted colony number was decreased in the presence of LBB, EG and CO. Significant results: Serum vs. E2 ("P=0.01); E2 vs. E2+LBB ( $\left.{ }^{* *} \mathrm{P}=0.02\right)$; E2 vs. E2+EG $\left({ }^{* *} \mathrm{P}=0.02\right)$; $\mathrm{E} 2$ vs. $\mathrm{E} 2+\mathrm{CO}\left({ }^{* *} \mathrm{P}=0.02\right) . \mathrm{AI}$, anchorage-independent; $\mathrm{E} 2$, $17 \beta$-estradiol; LBB, Lycium barbarum bark; EG, Epimedium grandiflorum; $\mathrm{CO}$, Cornus officinalis.

phenotypes must be further characterized at the molecular level to confirm the functional status of ER.

The comparative dose-response experiments of the herbal extracts on the ER-NF and ER-F phenotypes identified the $\mathrm{IC}_{50}$ values of the individual extracts independently for each phenotype, and the $\mathrm{IC}_{50}$ ratio of the ER-NF vs. the ER-F phenotype facilitated the determination of preferential efficacy and rank ordering of the Chinese nutritional herbs on isogenic MCF-7 phenotypes with modulated ER function. The rank order of growth-inhibitory activity based on the individual ER-NF:ER-F $\mathrm{IC}_{50}$ ratios revealed that the extracts from five herbs (LBB, EU, LBF, PC and DA) were preferentially effective against ER-F cells, while the extracts from four herbs (LL, CS, EG and LC) were equally effective against ER-NF and ER-F cells, and the extracts from two herbs (AS and CO) were preferentially effective against ER-NF cells. Thus, the data on differential susceptibility for growth inhibition on isogenic cells with modulated ER function provide potential leads for the efficacy of Chinese nutritional herbs on $\mathrm{ER}^{+}$and ER clinical breast cancer.

The $\mathrm{IC}_{90}$ values distinguished the maximum cytostatic response from the toxic response. Therefore, the $\mathrm{IC}_{90}$ values for the ER-NF and ER-F phenotypes were used for the mechanistic biomarker experiments on AI growth, cell cycle progression, cellular apoptosis and cellular E2 metabolism.

Selected herbal extracts representing specific phenotype-dependent preference for growth inhibition were also effective in inhibiting the AI growth of the ER-NF phenotype. Thus, at their respective $\mathrm{IC}_{90}$ value, these extracts induced a $>80 \%$ reduction in the number of AI colonies, relative to those observed in the E2-treated control group.

To identify the potential mechanisms responsible for their growth-inhibitory efficacy, the extracts from LBB (preferential activity against the ER-F phenotype), EG (equally effective in ER-NF and ER-F cells) and CO (preferential activity against the ER-NF phenotype) were examined for their effects on cell cycle progression and cellular apoptosis. These extracts exhibited 
Table VI. Effects of extracts from LBB, EG and CO on the cell cycle progression of the estrogen receptor non-functional phenotype.

Cell cycle-phase population $(\%)^{\mathrm{a}}$

\begin{tabular}{lcccc}
\cline { 3 - 4 } Treatment & Concentration & G1 & $\mathrm{S}$ & $\mathrm{G} 2 / \mathrm{M}$ \\
\hline E2 & $20 \mathrm{nM}$ & $50.1 \pm 5.8$ & $34.2 \pm 3.9$ & $14.9 \pm 1.6$ \\
LBB & $0.05 \%$ & $42.2 \pm 4.9$ & $17.7 \pm 1.9^{\mathrm{c}}$ & $38.1 \pm 1.8^{\mathrm{d}}$ \\
EG & $0.80 \%$ & $77.7 \pm 7.8^{\mathrm{b}}$ & $19.8 \pm 1.9^{\mathrm{c}}$ & $2.4 \pm 0.2^{\mathrm{e}}$ \\
CO & $0.10 \%$ & $66.9 \pm 6.7^{\mathrm{b}}$ & $18.5 \pm 1.8^{\mathrm{c}}$ & $14.6 \pm 1.4$
\end{tabular}

${ }^{\mathrm{a}}$ Determined by flow cytometry after a 48 -h. treatment. Data are represented as the mean $\pm \mathrm{SD}\left(\mathrm{n}=3 /\right.$ treatment group). ${ }^{\mathrm{b}} \mathrm{P}=0.04 \mathrm{vs}$. $\mathrm{E} 2,{ }^{\mathrm{c}} \mathrm{P}=0.04 \mathrm{vs}$. $\mathrm{E} 2$, ${ }^{\mathrm{d}} \mathrm{P}=0.01$ vs. E2, ${ }^{\mathrm{e}} \mathrm{P}=0.02$ vs. E2. E2, 17ß-estradiol; LBB, Lycium barbarum bark; EG, Epimedium grandiflorum; CO, Cornus officinalis.

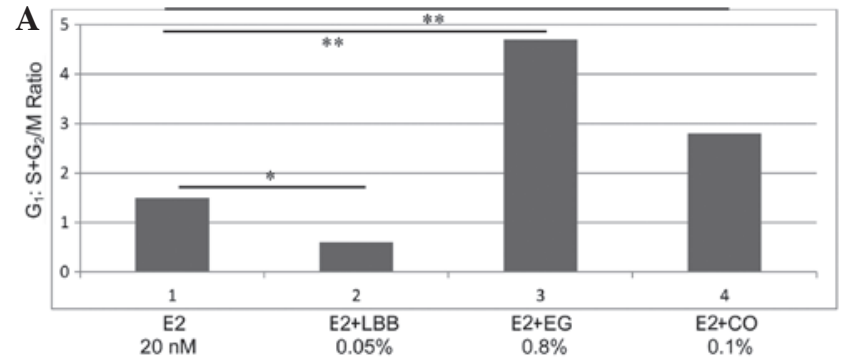

B

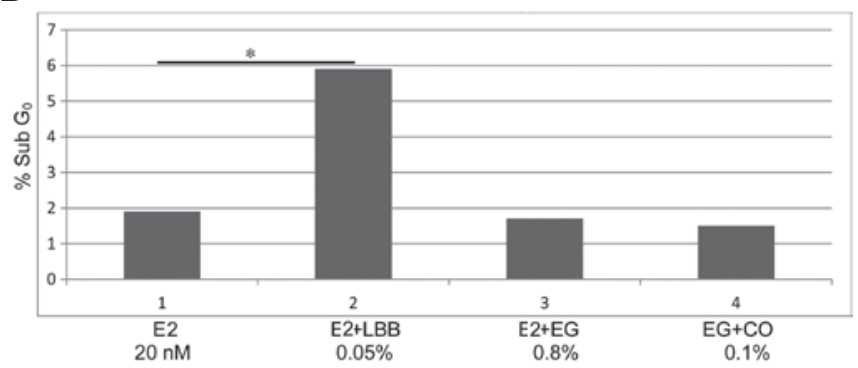

Figure 3. (A and B) Modulation of cell cycle progression and induction of cellular apoptosis. (A) Effects of extracts from LBB, EG and CO on the $\mathrm{G} 1: \mathrm{S}+\mathrm{G} 2 / \mathrm{M}$ ratio. Treatment with the extract from LBB decreased this ratio $\left({ }^{*} \mathrm{P}=0.04\right)$ due to $\mathrm{G} 2 / \mathrm{M}$ arrest, while treatment with the extracts from $\mathrm{EG}$ $\left({ }^{* *} \mathrm{P}=0.02\right)$ and $\mathrm{CO}\left({ }^{* *} \mathrm{P}=0.02\right)$ increased the above ratio due to $\mathrm{G} 1$ arrest. (B) Effects of extracts from LBB,EG and CO on induction of cellular apoptosis Only the extract from LBB effectively induced cellular apoptosis ( $\mathrm{P}=0.01)$. E2, 17 $\beta$-estradiol; LBB, Lycium barbarum bark; EG, Epimedium grandiflorum; $\mathrm{CO}$, Cornus officinalis.

distinct effects on cell cycle progression. Thus, the LBB extract induced G2/M arrest and cellular apoptosis, while the extracts from EG and $\mathrm{CO}$ induced $\mathrm{G} 1$ arrest but were ineffective regarding the induction of cellular apoptosis. Thus, extracts from effective herbs representative of each of the aforementioned three groups exhibit distinct effects on cell cycle progression and/or cellular apoptosis. Taken together, these data suggest that the anti-proliferative activity of the above three herbal extracts may be due to their distinct effect on specific phases of the cell cycle.

Oxidative metabolism of E2 impacts the carcinogenesis of endocrine-responsive target organs (19-22). E2 metabolites such as 2-OHE1 and 16 $\alpha$-OHE1 exhibit distinct growth-modulatory effects on mammary epithelial cells at the initial phases of carcinogenesis induced by certain oncogenes such as RAS, c-Myc and HER-2, as well as on

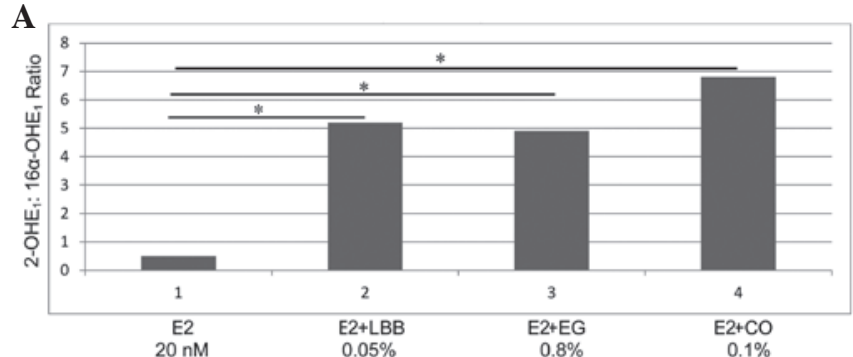

B

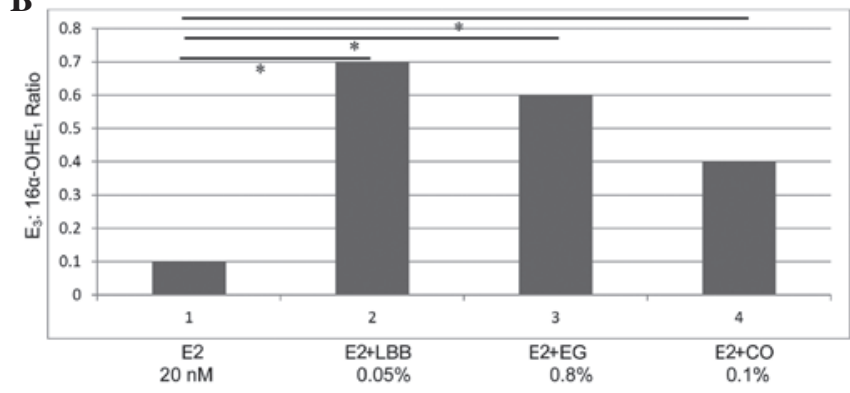

Figure 4. (A and B) Alteration in the cellular metabolism of E2. (A) Effect of extracts from LBB, EG and CO on the 2-OHE1:16 $\alpha$-OHE1 ratio. An increase in 2-OHE1:16 $\alpha$-OHE1 ratio was observed in response to treatment with the three extracts, relative to the ratio exhibited by the E2-treated control ( $\mathrm{P}=0.01$ ). (B) Effect of extracts from LBB, EG and CO on the $\mathrm{E} 3: 16 \alpha$-OHE1 ratio. An increase in $\mathrm{E} 3: 16 \alpha-\mathrm{OHE} 1$ ratio $\left({ }^{*} \mathrm{P}=0.01\right)$ in response to treatment with the three extracts was observed, relative to that displayed by the E2-treated control. E2, 17ß-estradiol; LBB, Lycium barbarum bark; EG, Epimedium grandiflorum; CO, Cornus officinalis; 2-OHE1; 2-hydroxyestrone; 16 $\alpha$-OHE1, 16 $\alpha$-hydroxyestrone; E3, estriol.

breast carcinoma-derived cells (22-25). Thus, 2-OHE1 exhibits anti-proliferative effects on these cells, while 16 $\alpha$-OHE1 promotes cellular proliferation (22-25). Experimentally induced alterations in E2 metabolism, measured as 2-OHE1:16 $\alpha$-OHE1 and E3:16 $\alpha$-OHE1 ratios, provide an endocrine biomarker for carcinogenic risk and for effective cancer prevention/therapy $(10,12,13)$. In the light of these observations, the effects of extracts from LBB, EG and CO on E2 metabolism were examined in the ER-NF phenotype of MCF-7 cells. The data from these experiments demonstrated that these three extracts at their respective $\mathrm{IC}_{90}$ value increased the 2-OHE1:16 $\alpha$-OHE1 and E3:16 $\alpha$-OHE1 ratios due to enhanced production of the anti-proliferative metabolites 2-OHE1 and E3. 
The outcome of the present study demonstrates that non-fractionated aqueous extracts from Chinese nutritional herbs effectively down-modulate the growth-promoting effects of E2 via distinct mechanisms in the isogenic cell culture model with modulated ER function. It is noteworthy that human breast carcinoma-derived cell culture models with differing expression of ER, PR, HER-2, epidermal growth factor receptor, p53 and retinoblastoma protein have been utilized to examine the growth-inhibitory effects of aqueous extracts prepared from several Chinese medicinal herbs $(26,27)$. However, it should be recognized that intrinsic genetic differences in expression of hormone and growth factor receptors in individual cell lines preclude justifiable comparative investigations within the existing models. By contrast, the data from the present study provide evidence that isogenic phenotypes with modulated ER function as the only experimental variable represent a facile comparative approach for a mechanism-based screening of extracts prepared from multi-functional herbs. Furthermore, rank ordering of preferentially efficacious herbal extracts on isogenic ER-NF and/or ER-F phenotypes may provide valuable mechanistic leads for their potential efficacy towards $\mathrm{ER}^{-}$and/or $\mathrm{ER}^{+}$clinical breast cancer.

It must be emphasized that, in the present study, the non-fractionated aqueous extracts from the herbs were used specifically to simulate the administration of herbal tea to patients in traditional Chinese medicine (4,5,7). It is therefore conceivable that a mixture of water soluble bio-active agents may be responsible for the observed growth-inhibitory effects in the present experimental models, and that these agents, acting individually or in combination, may be affecting the growth of MCF-7 cells.

It is also noteworthy that the parental MCF-7 cells, which represent a model for the luminal A molecular subtype of clinical breast cancer, have been previously tested as a model for endocrine therapy-resistant cancer stem cells (28). The response of MCF-7-derived stem cells to nutritional herbs may therefore identify a testable approach for efficacious cancer stem cell-targeted herbal therapy. Indeed, efficacy of natural phytochemicals such as quercetin and sulforaphane towards pancreatic cancer stem cells has been documented (29).

In conclusion, the present study demonstrates that the non-fractionated aqueous extracts from Chinese nutritional herbs effectively down-modulate the growth-promoting effects of E2 via distinct mechanisms in the present isogenic cell culture model. This aspect is specifically strengthened by the data that extracts from herbs selectively efficacious for the ER-F or ER-NF phenotype function via distinct effects on cell cycle progression, cellular apoptosis and formation of anti-proliferative E2 metabolites. Furthermore, the models developed and the results obtained in the present study provide relevant significance to the herbal management of $\mathrm{ER}^{+}$or $\mathrm{ER}^{-}$clinical breast cancer, as well to the relapse of $\mathrm{ER}^{+}$breast cancer, in which the ER expression has become negative $(4,5,26,27,30)$. Clearly, clinical usefulness of the nutritional herbs investigated in the present study will have to be established in future human studies.

\section{Acknowledgements}

Major funding for the present study was provided by philanthropic contributions to the American Foundation for Chinese
Medicine (New York, NY, USA) by the family of Mr. Daniel and Ms. Kathleen Mezzalingua, the family of Mr. Hakan and Ms. Marie Ledin, the Laura and Isaac Perlmutter Foundation Inc. (Lake Worth, FL, USA), the Saint Agatha Foundation (Jenkintown, PA, USA) and the Sophie Stenbeck Family Foundation (New York, NY, USA).

\section{References}

1. Lippman ME: Efforts to combine endocrine and chemotherapy in the management of breast cancer: Do two and two equals three? Breast Cancer Res Treat 3: 117-127, 1983.

2. Johnston SR and Dowsett M: Aromatase inhibitors for breast cancer: Lessons from the laboratory. Nat Rev Cancer 3: 821-831, 2003.

3. Musgrove EA and Sutherland RL: Biological determinants of endocrine resistance in breast cancer. Nat Rev Cancer 9: 631-643, 2009.

4. Rock E and De Michelle A: Nutritional approaches to late toxicities of adjuvant chemotherapy in breast cancer survivors. J Nutr 133 (Suppl 1): S3785-S3793, 2003.

5. Heyler LK, Chin S, Chu BK, Fitzgerald B, Verma S, Rakovitch E, Dranitsaris $\mathrm{G}$ and Clemons M: The use of complementary and alternative medicine among patients with locally advanced breast cancer: A descriptive study. BMC Cancer 6: 39, 2006.

6. Oudin C, Bonnetain F, Boidot R, Vegran F, Soubeyrand MS, Arnould L, Riedinger JM and Lizard-Nacol S: Patterns of loss of heterozygosity in breast carcinoma during neoadjuvant chemotherapy. Int J Oncol 30: 1145-1151, 2007.

7. Mathews AK, Sellergren SA, Huo D, List M and Fleming G: Complementary and alternative medicine use among breast cancer survivors. J Alt Comp Med 13: 555-562, 2007.

8. Lippman ME, Osborne CK, Knazek R and Young N: In vitro model systems for the study of hormone-dependent breast cancer. N Engl J Med 296: 154-159, 1977.

9. Suto A, Bradlow HL, Kubota T, Kitajima H, Wong GY, Osborne MP and Telang NT: Alteration in proliferative and endocrine responsiveness of human mammary carcinoma cells by prototype tumor suppressing agents. Steroids 58: 215-219, 1993.

10. Li G, Sepkovic DW, Bradlow HL, Telang NT and Wong GYC: Lycium barbarum inhibits growth of estrogen receptor positive human breast cancer cells by favorably altering estradiol metabolism. Nutr Cancer 61: 408-414, 2009.

11. Mukherjee B, Telang N and Wong GY: Growth inhibition of estrogen receptor positive human breast cancer cells by Taheebo from the inner bark of Tebebuia avellandae tree. Int J Mol Med 24: 253-260, 2009.

12. Telang NT, Li G, Sepkovic DW, Bradlow HL and Wong GY: Anti-proliferative effects of Chinese herb Cornus officinalis in a cell culture model for estrogen receptor positive clinical breast cancer. Mol Med Rep 5: 22-28, 2012.

13. Telang N, Li G, Sepkovic D, Bradlow HL and Wong GY: Comparative efficacy of extracts from Lycium barbarum bark and fruit on estrogen receptor positive human mammary carcinoma MCF-7 cells. Nutr Cancer 66: 278-284, 2014.

14. Katdare M, Osborne M and Telang NT: Soy isoflavone genestein modulates cell cycle progression and induces apoptosis in HER-2/neu oncogene expressing human breast epithelial cells. Int J Oncol 21: 809-815, 2002.

15. Sorlie T, Perou CM, Tibshirani R, Aas T, Geisler S, Johnsen H, Hastie T, Eisen MB, van de Rijn M, Jeffrey SS, et al: Gene expression patterns of breast carcinomas distinguish tumor subclasses with clinical interpretations. Proc Natl Acad Sci USA 98: 10869-10874, 2001.

16. Neve RM, Chin K, Fridyand J, Yeh J, Baehner FL, Fevr T, Clark L, Bayani N, Coppe JP, Tong F, et al: A collection of breast cancer cell lines for the study of functionally distinct cancer subtypes. Cancer Cell 10: 515-527, 2006.

17. Subik K, Lee JF, Baxter L, Strzepak T, Costello D, Crowley P, Xing L, Hung MC, Bonfiglio T, Hicks DG and Tang P: The expression patterns of ER, PR, HER-2, CK5/6, EGFR, Ki 67 and AR by immunohistochemical analysis in breast cancer cell lines. Breast Cancer (Auckl) 4: 35-41, 2010.

18. Muller PA and Vousden KH: Mutant p53 in cancer: New functions and therapeutic opportunities. Cancer Cell 25: 304-317, 2014.

19. Schneider J, Kinne D, Frachia A, Pierce V, Anderson KE, Bradlow HL and Fishman J: Abnormal oxidative metabolism of estradiol in women with breast cancer. Proc Natl Acad Sci USA 79: 3047-3051, 1982. 
20. Fishman J, Schneider J, Herschkopf RJ and Bradlow HL: Increased estrogen 16alpha-hydroxylase activity in women with breast and endometrial cancer. J Steroid Biochem 20: 1077-1081, 1984.

21. Yager JD and Davidson NE: Estrogen carcinogenesis in breast cancer. N Engl J Med 354: 270-282, 2006.

22. Telang NT: Oncogenes, estradiol biotransformation, and mammary carcinogenesis. Ann NY Acad Sci 784: 277-287, 1996.

23. Suto A, Telang NT, Tanino H, Takeshita T, Ohmiya H, Osborne MP and Kubota T: In vitro and in vivo modulation of growth regulation in the human breast cancer cell line MCF-7 by estradiol metabolites. Breast Cancer 6: 87-92, 1999.

24. Lottering ML, Haag M and Segers JC: Effects of 17beta-estradiol metabolites on cell cycle events in MCF-7 cells. Cancer Res 52: 5926-5932, 1992.

25. Schneider J, Huh MM, Bradlow HL and Fishman J: Antiestrogen action of 2-hydroxyestrone on MCF-7 human breast carcinoma cells. J Biol Chem 259: 4840-4845, 1984.

26. Campbell MJ, Hamilton B, Shoemaker M, Tagliaferri M, Cohen I and Tripathy D: Anti-proliferative activity of Chinese medicinal herbs on breast cancer cells in vitro. Anticancer Res 22: 3843-3852, 2002.
27. Chiu JH, Chang C, Wu JC, Liu HJ, Wen CS, Hsu CH, Chen JL, Tseng LM, Chen WS and Shyr YM: Screening to identify commonly used Chinese herbs that affect ERBB2 and ESR1 gene expression using the human breast cancer MCF-7 cell line. Evid Based Complement Alternat Med 2014: 965486, 2014.

28. Telang N: Putative cancer-initiating stem cells in cell culture models for molecular subtypes of clinical breast cancer. Oncol Lett 10: 3840-3846, 2015

29. Zhou W, Kallifatidis G, Baumann B, Rausch V, Mettern J Gladkich J, Giese N, Moldenhauer G, Wirth T, Büchler MW, et al: Dietary polyphenol quercetin targets pancreatic cancer stem cells. Int J Oncol 37: 551-561, 2010.

30. Ye L, Jia Y, Ji KE, Sanders AJ, Xue K, Ji J, Mason MD and Jiang WG: Traditional Chinese medicine in the prevention and treatment of cancer and cancer metastasis. Oncol Lett 10: $1240-1250,2015$. 